\title{
Hierarchical Matching Beats The Non-Wildcard and Interpretation Tree Model Matching Algorithms
}

\author{
Robert B. Fisher \\ Dept. of Artificial Intelligence, University of Edinburgh \\ 5 Forrest Hill, Edinburgh EH1 2QL, Scotland, United Kingdom
}

\begin{abstract}
In Fisher[1] we introduced a non-wildcard model matching algorithm that has speed advantages over the standard Interpretation Tree model matching algorithm. This paper describes a hierarchical model-matching algorithm that has improved performance over both the standard and non-wildcard algorithms.
\end{abstract}

\section{Introduction}

The most well-known control algorithm for high-level model matching in computer vision is the Interpretation Tree(IT) expansion algorithm, as used by Grimson and Lozano-Perez[2, 3]. In Fisher[1] we introduced a variation on this algorithm that did not use a wildcard which gave performance advantages of 4-10. Both algorithms search a tree of model-to-data correspondences, such that each node in the tree represents one correspondence and the path of nodes from the current node back to the root of the tree is a set of simultaneous pairings. The non-wildcard algorithm avoids the many matches requiring wildcards and only investigates the single model-to-data pairings once, while still exploring the same match search space. It works by extending a set of matches by only adding pairs of matching real model-to-data pairings, rather than also adding pairings that contain wildcards. These algorithms both have the potential for combinatorial search explosion, hence prompting further research into alternative algorithms.

The main cause of the complexity is the re-exploration of the same search subspaces on back-tracking to consider new initial matches. This paper describes a hierarchical model-matching algorithm that results in improved performance over both the standard and non-wildcard algorithms, over a wide range of problem conditions. In the discussion that follows, the term generated refers to matches that are hypothesized prior to consistency testing, and accepted refers to matches that pass the consistency tests. The following quantities are used:

- There are $M$ model features in the model.

- On average, $p_{v} M$ of these are visible in the scene (e.g. less than $M$ by occlusion). In $2 \mathrm{D}$ scenes, $p_{v} \doteq 1$ and, in $3 \mathrm{D}$ scenes, $p_{v} \stackrel{\doteq}{=} 0.5$ as about half of the features are on the back side of the object and hence not visible.

- Of the visible model features, only $p_{r}$ of these are recognizable (because of segmentation failures, etc.) forming $C=p_{r} p_{v} M$ correct matchable data features. (If the model chosen for this scene is incorrect, $p_{r}=0$.) Which $C$ of the $M$ model features are matchable is not known initially. 


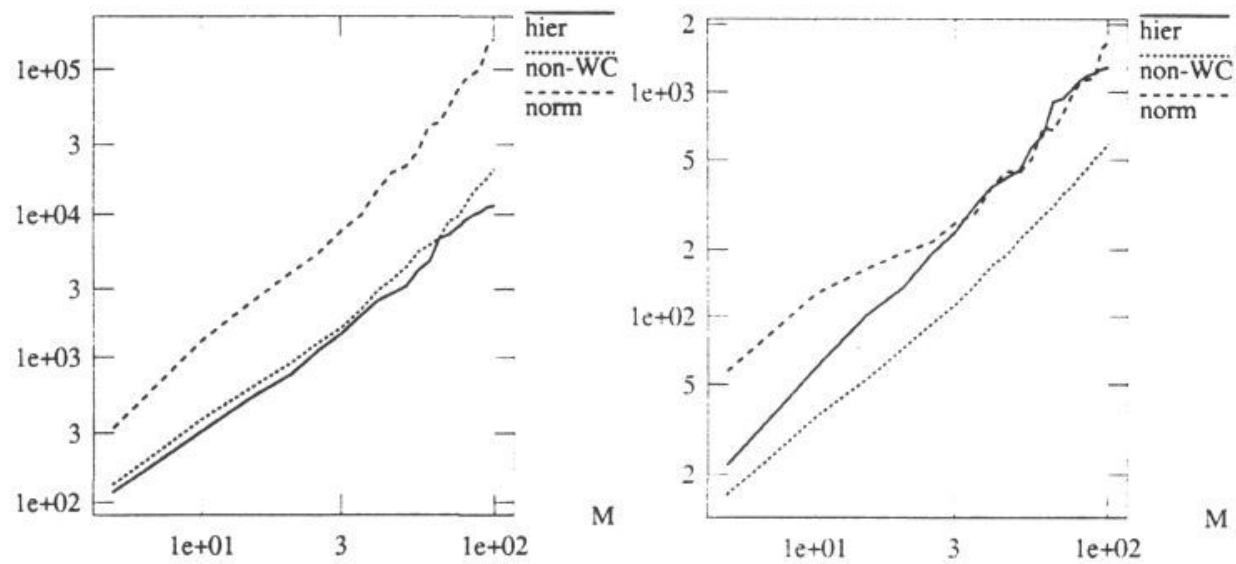

Figure 1: Generated and Accepted Nodes versus Number of Model Features $(M)$ with $S=20 p_{r}=0.95 p_{1}=0.1 p_{2}=0.01 p_{v}=0.5 \tau=0.5$ (loglog plot)

- There are also $S$ spurious data features (e.g. noise features and unrecognizable visible model features). Altogether there are $D=C+S$ data features.

- The probability that a randomly chosen model feature matches with an incorrect random data feature is $p_{1}$ (correct pairings alway match).

- The probability that a random pair of model features is consistent with an incorrect random pair of data features (given that the individual model-todata pairings are consistent) is $p_{2}$.

- An acceptable set of model-to-data pairings must have at least $T=\tau p_{v} M$ non-wildcard correspondences $(\tau \in[0 . .1])$. Whenever this many are achieved, then the whole matching process terminates successfully immediately. Any set of matches that can never get $T$ matches (because insufficient potential matches remain) is terminated immediately and the matching process proceeds to considering other matches.

\section{The Hierarchical Matching Algorithm}

Suppose that the $M=K^{L}$ model features can be decomposed into $K^{L-1}$ submodels each containing $K$ features. Each of these submodels are grouped into $K^{L-2}$ larger submodels containing $K^{2}$ features, and so on hierarchically until there is one top-level model containing all $K^{L}$ model features. The matching algorithm described below generates hypotheses of these submodel types, and only these submodels can be matched together to create hypotheses of the next larger model type.

We describe here a binary submodel hierarchy matching algorithm (i.e. $K=2$ ). As an example, consider the simple model hierarchy consisting of four model features $A-D$ organized into two larger submodels $A B$ and $C D$, which are combined into a larger model $A B C D$. We use a top-down matching strategy (e.g. look for instances of $A B C D$, which recursively looks for instances of $A B$ and $C D$, etc.). Then, at level $\lambda$ from the bottom of the model hierarchy, we need only compare the hypotheses that were successfully generated at level $\lambda-1$. 


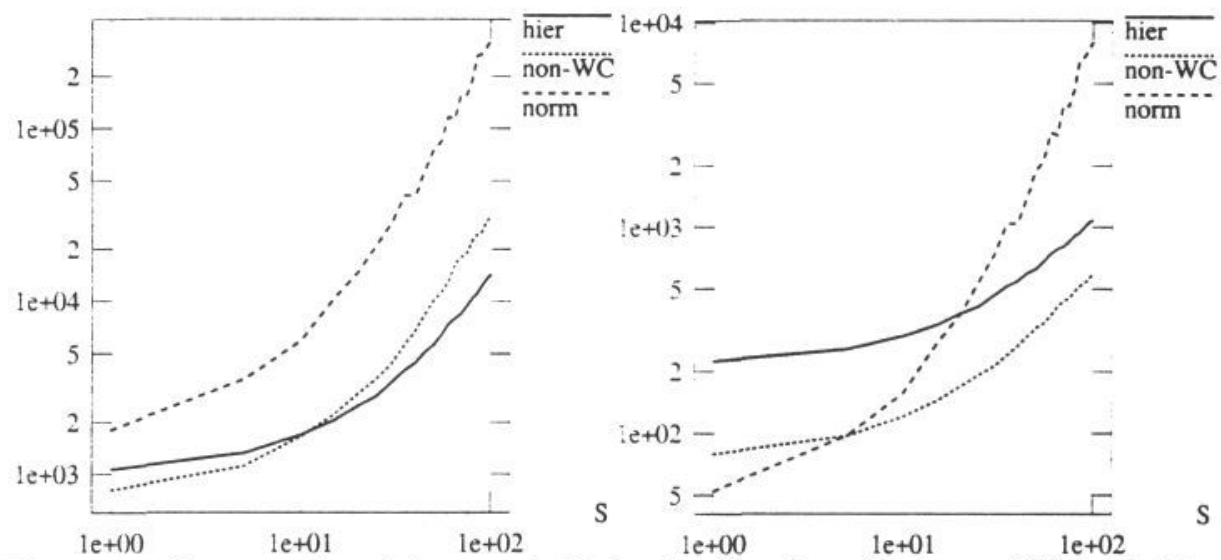

Figure 2: Generated and Accepted Nodes by Spurious Features $(S)$ with $M=$ $40 p_{r}=0.95 p_{1}=0.1 p_{2}=0.01 p_{v}=0.5 \tau=0.5$ (loglog plot)

The algorithm records hypotheses accepted at any given point in the model hierarchy. Thus, if the algorithm needs to backtrack from level $A B C D$ to find a new hypothesis for $A B$, it is not necessary to later re-explore previously explored portions of the matching space (e.g.) for $C D$. The algorithm recalls and retries previously verified matches, and then, if there were no successes, generates additional hypotheses starting from where the matching last stopped for this model. This provides a substantial savings over the standard IT algorithm.

At the lowest levels of the hierarchy, many consistent hypotheses are composed mainly of wildcards. Hence, the hierarchical algorithm uses a "largestsubhypothesis-first" search algorithm (i.e. having the most matched non-wildcard data features). The algorithm determines the largest possible hypothesis size, then attempts to generate hypotheses of that size before considering smaller hypotheses. It matches together subhypotheses that may be of different sizes, but tries the largest ones first. The algorithm for pairing hypotheses at this level uses a sensible ordering: (1) all hypotheses that have $N$ matched data features are generated before any hypothesis having $N-1$ and (2) amongst all hypotheses with the same size, the algorithm generates the hypotheses in the order that keeps the subhypothesis sizes as similar as possible (e.g. it chooses the pair of sizes $(3+2)$ over the pair of sizes $(4+1)$ )

At higher levels, when a new subhypothesis is needed, the algorithm may: (1) return a previously generated subhypothesis of the desired size, (2) generate a new subhypothesis that is the same size as that returned at the last call (if there was a previous call), (3) generate the largest possible new subhypothesis of a smaller size or (4) fail when no more subhypotheses are possible.

When the recursive request for hypotheses reaches the lowest level, (e.g. model feature $A$ in the above example), the algorithm matches the original data features to the original primitive model submodels. New pairings are tested on demand from above, so not all given model-data feature pairings need always be tested before a successful match is found. Consistency is tested using the standard unary feature matching tests. After all possible matches with data features have been attempted, then a match using the wildcard is generated. This promotes filled 


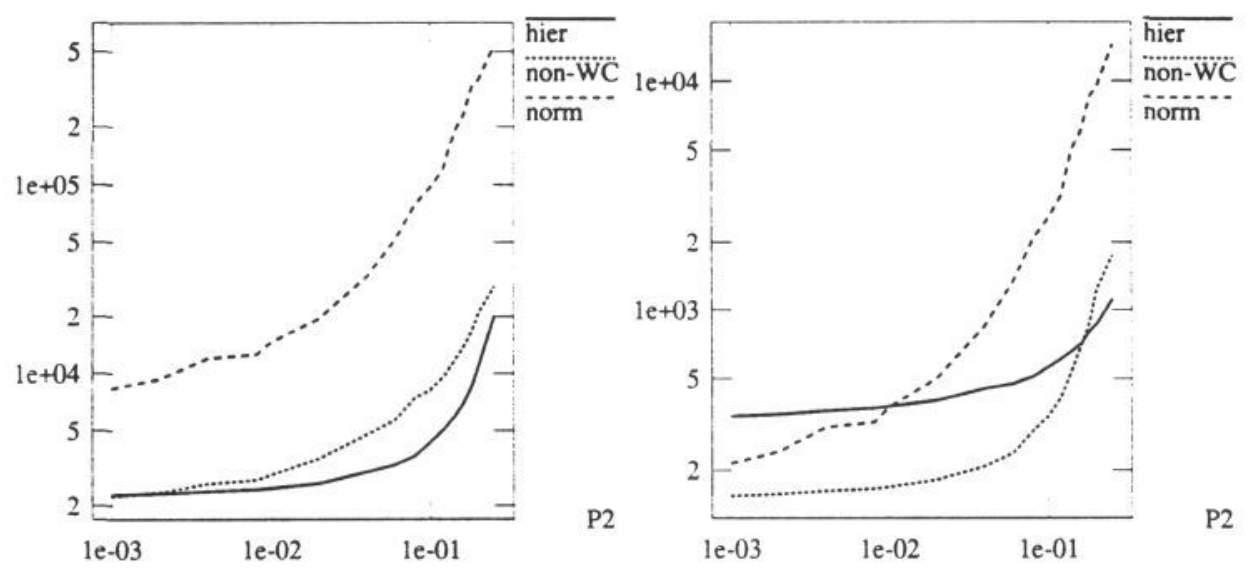

Figure 3: Generated and Accepted Nodes by Binary Match Probability $\left(p_{2}\right)$ with $M=40 S=20 p_{r}=0.95 p_{1}=0.1 p_{v}=0.5 \tau=0.5$ (loglog plot)

hypotheses over the proliferation of empty hypotheses.

When testing subhypothesis consistency at level 1 (i.e. pairings involving two model and data features), the standard algorithm's binary feature matching tests are used. At level 2 and higher, consistency is based on the same binary compatibility tests, only tested using primitive model-data feature pairings that come from different subhypotheses.

This algorithm works because, by induction on the previous level, the subhypotheses generated for the two submodels at the next lower level are also recursively generated in a largest-first order, and are then combined in the order that produces the largest hypotheses first. The order of features in the hierarchy is not important to the success of the algorithm, and the algorithm does not require the model to have any natural binary decomposition. However, efficiency is improved if highly likely matches are in the leftmost nodes, thus preventing the algorithm from having to back-track through false starts. Appendix A gives pseudocode for this algorithm and Appendix B gives an example of a matching.

\section{The Experiments}

To demonstrate the effectiveness of the hierarchical search algorithm, as compared to the non-wildcard and standard algorithms, we use the following simulated experimental problem, based on an example described in Grimson[4]. (A real problem follows.) Grimson showed that the model and simulation gave a reasonable characterization of real matching problems. The use of the simulated problems then allows us to compare the algorithm performance on data sets of varying sizes.

Based on the problem model given in Section 2, each model-match experiment of the three algorithms will consist of:

1. Initially determining a random selection of $C$ of the $D$ data features to be the solution. 


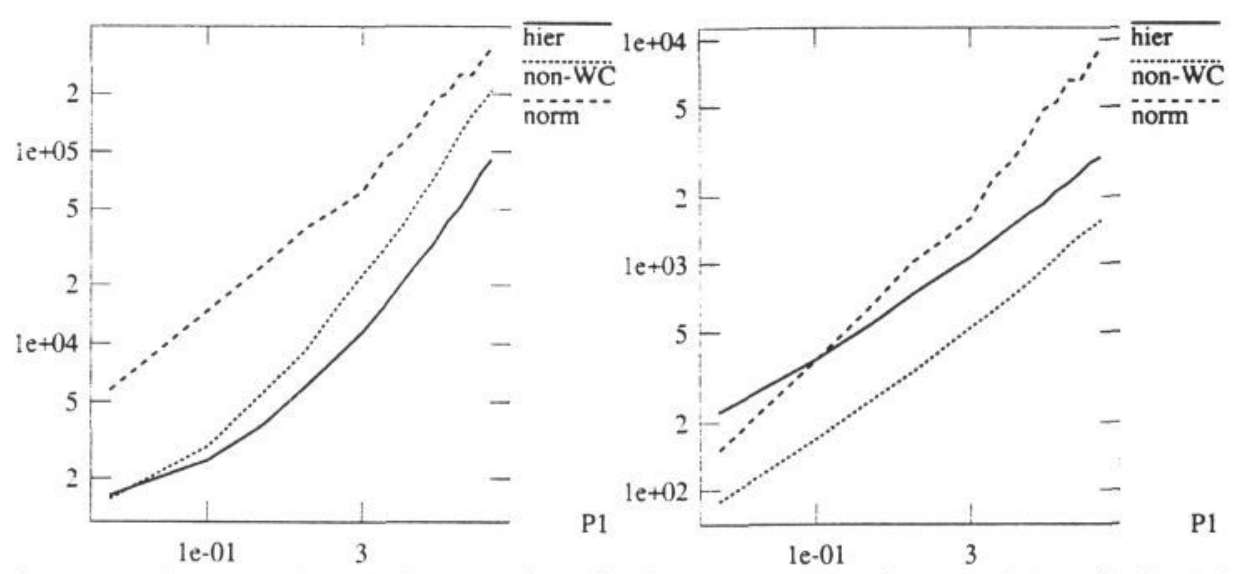

Figure 4: Generated and Accepted Nodes by Unary Match Probability $\left(p_{1}\right)$ with $M=40 S=20 p_{r}=0.95 p_{2}=0.01 p_{v}=0.5 \tau=0.5$ (loglog plot)

2. For each generated model-to-data pairing, a correspondence that is not part of the solution and does not use a wildcard is accepted if the new correspondence is individually satisfied with probability $p_{1}$ and the new correspondence is pairwise satisfied with each previously filled non-wildcard feature with probability $p_{2}$. Correspondences that are part of the solution or use the wildcard are always accepted.

For the experiments described in this paper, we used:

\begin{tabular}{|l|c|l|}
\hline PARAMETER & NOMINAL & RANGE \\
\hline$M$ & 40 & 5 to 100 by 5 \\
\hline$S$ & 20 & 0 to 100 by 5 \\
\hline$p_{1}$ & 0.1 & 0.05 to 0.75 by 0.05 \\
\hline$p_{2}$ & 0.01 & $\begin{array}{l}0.001,0.002,0.004,0.008,0.01, \\
\end{array}$ \\
\hline$\tau$ & 0.02 to 0.20 by $0.02,0.25$ \\
\hline$p_{v}$ & 0.5 & 0.2 to 0.9 by 0.1 \\
\hline$p_{r}$ & 0.95 & no variation \\
\hline
\end{tabular}

In each experiment described in this section, one parameter was varied over the range given above and all others were set to the nominal value. All experiments were run 200 times and the value reported is the mean value. The graphs in Figures 1-5 given show how the number of nodes generated and accepted varied with the parameters for the hierarchical, non-wildcard and standard IT algorithms. The results for the non-wildcard algorithm are an improvement on those given in [1], due to improvements in the algorithm. As we look over the results, which explore a substantial portion of the parameter spaces likely to be encountered in visual matching problems, we can see that the hierarchical algorithm is better than the non-wildcard and standard algorithm with respect to the number of nodes searched except when $\tau$ becomes large, but the non-wildcard algorithm is better with regards to the number of nodes accepted except for when $p_{2}$ becomes large.

When there is no instance of the object in the scene, it is unlikely that the early success conditions would occur, and thus almost all of the search space would have 

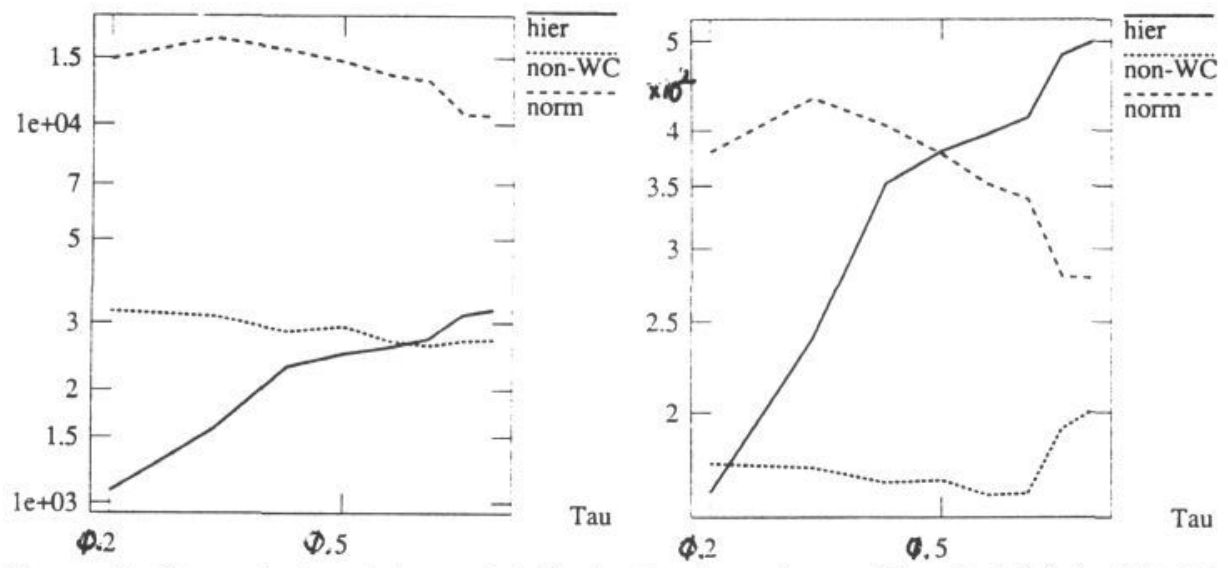

Figure 5: Generated and Accepted Nodes by Acceptance Threshold $(\tau)$ with $M=$ $40 S=20 p_{r}=0.95 p_{1}=0.1 p_{2}=0.01 p_{v}=0.5$ (loglog plot)

to be explored. In this case, a much greater amount of work is required. In the case of the hierarchical algorithm, about 17 times more work is required to reject a match in a scene, but this is still 4 times better than the standard algorithm (but requires about twice the work of the non-wildcard algorithm).

The algorithms were compared on edges extracted from real test scene similar to those used by Grimson. Because the algorithms are sensitive to data feature order, the algorithms were run 100 times with the model and data features permuted randomly. The effective probabilities in this scene were $p_{1}=.235$ and $p_{2}=0.017$ and the number of features were $M=13$ and $D=129$. Seven of 13 model edges match true data edges in the test scene using the given tolerances. The average time taken for the matching algorithms on a Sparcstation 1+ was 1.47 seconds for the hierarchical algorithm and $5.88 \mathrm{sec}$. for the standard algorithm (and 0.96 sec. for the non-wildcard algorithm). The mean number of nodes generated and accepted was 55025 and 1721 for the hierarchical algorithm, 64412 and 845 for the non-wildcard algorithm and 544171 and 39711 for the standard algorithm. On another test scene containing 10 instances of one of these parts, the average times required for a match was hierarchical $21.4 \mathrm{sec}$., non-wildcard $20.4 \mathrm{sec}$. and standard $419 \mathrm{sec}$. The effective probabilities in this scene were $p_{1}=.288$ and $p_{2}=0.011$ and the number of features were $M=28$ and $D=191$. The extra memory costs of recording the successful submatches in the hierarchical algorithm was about $1 \mathrm{M}$ bytes.

\section{Discussion and Conclusions}

Based on the simulated matchings, the hierarchical algorithm searches about 10 times fewer nodes than the standard algorithm and about one-half the nodes of the non-wildcard algorithm (as the number of features matched grows). However, it is also a much more complicated algorithm and thus the computational costs per search step are higher and it also executes more (expensive) binary tests (53237 vs 43682 for the non-wildcard algorithm in the first real-data test) before hypothesis rejection. In the two real examples cited in the previous section, the hierarchi- 
cal algorithm achieved a performance gain of about 4 to 20 over the standard algorithm, but was comparable in speed to the non-wildcard algorithm. However, based on the simulated results, it is clear that the performance of the algorithms may vary greatly on any one data set. It is also the case that the choice between the hierarchical and non-wildcard algorithms depends on the problem parameters. In particular, when $S, p_{1}$ or $p_{2}$ are large or $\tau$ is small, there are advantages to the hierarchical algorithm.

On the other hand, there are also some general guidelines when the hierarchical algorithm is not as effective as the non-wildcard algorithm. One general principle is "avoid proliferating reasonable hypotheses at the lowest levels of the matching". If we are analyzing a scene with many instances of the same object, or a scene where there are many nearly possible matches, or symmetry, then the hierarchical algorithm will generate many valid matches at the lowest levels, and they will all be explored until the first successful match is found. In a sense, the algorithm is simultaneously finding all matches, of which only one is needed. In addition, the non-wildcard algorithm generally accepts about one-half the number of nodes as the hierarchical algorithm. So, if the costs of processing an accepted node are high, the non-wildcard algorithm is to be favored. However, our experience with the real image data suggests that it is the generation costs, and in particular the costs of the binary feature tests that determine the running speed of the algorithms. Hence, the hierarchical algorithm has clear advantages in this respect.

\section{Acknowledgements}

This research was funded by SERC (IED grant GR/F/38310). Other facilities provided by University of Edinburgh. This paper benefited greatly from discussions with D. Borges, A. Fitzgibbon, J. Hallam, H. Hughes, M. Orr, K. Simsarian, M. Waite, M. Trucco and M. Uschold.

\section{References}

[1] Fisher, R. B., Non-Wildcard Matching Beats The Interpretation Tree, Proc. 1992 British Machine Vision Association Conf., pp 560-569, Leeds, 1992.

[2] Grimson, W. E. L., Lozano-Perez, T., Model-Based Recognition and Localization from Sparse Range or Tactile Data, International Journal of Robotics Research, Vol. 3, pp 3-35, 1984.

[3] Grimson, W. E. L., Object Recognition By Computer: The Role of Geometric Constraints, MIT Press, 1990.

[4] Grimson, W. E. L., The Combinatorics of Heuristic Search Termination for Object Recognition in Cluttered Environments, Lecture Notes in Computer Science, ECCV-90, Springer-Verlag, pp 552-556, 1990.

\section{A Hierarchical Search Algorithm}


if all done, return fail

if treetop is a base level subtree

$\{$ if another untried data feature (generate wildcard last)

$\{$ increment generated count

if not wildcard and unary test fails

then skip this subhypothesis

record this consistent subhypothesis

increment accepted count

return success\}

else return fail\}

// special case of only the left subtree of tree used

if only left subtree used

$\{$ recurse on left subtree

if early success or fail then return code

record subhypothesis for future regeneration

return success\}

// normal recursive case

do \{

top:

if first attempt at finding this hypothesis

$\{$ recurse on left subtree

if early success or fail then return code

record subhypothesis for future regeneration

recurse on right subtree

if early success or fail then return code

record subhypothesis for future regeneration

reset regeneration pointers

go to testsection\}

// normal hypothesis generation

is there a previously generated hyp for slot 1?

yes, is it the required size?

yes: go to testsection

no: smaller, go to getnewsloto

no: recursively regenerate new slot 1

early success: return early success

normal success: record subhypothesis and go to top

fail: proceed to getnewsloto

getnewslot0:

is there a previously generated hyp for slot 0?

yes, is it the required size?

yes: reset slot 1 list for the current needed size

and go to testsection

no: go to getnextvalidsize

no: recursively regenerate new slot 0

early success: return early success

normal success:

record subhypothesis 
reset slot 1 list for the current needed size

if no slot 0 of needed size go to getnextvalidsize

else go to testsection

fail: proceed to getnextvalidsize

getnextvalidsize: // get new candidate subhypothesis sizes

do \{

get next smallest hypothesis size

if no more, then return fail

if size of both slots negative, then return fail

if either slot negative, then continue

if slot 0 size does not exist, then continue

if slot 1 size does not exist, then continue

reset slots 0 and 1 for use with this size

\} until a valid new size is found

testsection: // test this hypothesis

increment generated count

if neither subhypotheses are completely wildcards

$\{$ do binary tests between all non-wildcard features

of the first subhypothesis and all non-wildcard

features of the second subhypothesis

if failure, continue\}

// a consistent match

record subhypothesis for future regeneration

increment accepted count

if enough features matched then return early success

if cannot match enough features in remaining unfilled slots

then reject subhypothesis

return new match

\} forever \}

\section{B Example of Hierarchical Matching Algorithm}

Suppose we have the example $A B C D$ hierarchical model described in Section 2. Suppose also that the model features can make these individual matches against data features $\mathrm{a}, \mathrm{b}, \mathrm{c}$ and the wildcard $*$. We assume that the early termination criterion requires at least 3 features matched.

\begin{tabular}{|c|c|c|}
\hline $\begin{array}{c}\text { Model } \\
\text { Feature }\end{array}$ & $\begin{array}{c}\text { Data } \\
\text { Features }\end{array}$ & $\begin{array}{c}\text { True } \\
\text { Match }\end{array}$ \\
\hline $\mathrm{A}$ & $\mathrm{a},{ }^{*}$ & $\mathrm{a}$ \\
$\mathrm{B}$ & $\mathrm{b},{ }^{*}$ & $\mathrm{~b}$ \\
$\mathrm{C}$ & $\mathrm{c}^{*}$ & $\mathrm{c}$ \\
$\mathrm{D}$ & $\mathrm{a}^{*}$ & $*$ \\
$\mathrm{AB}$ & $\mathrm{ab}, \mathrm{a}^{*},{ }^{*} \mathrm{~b},{ }^{* *}$ & $\mathrm{ab}$ \\
$\mathrm{CD}$ & $\mathrm{ca}^{*} \mathrm{c}^{*},{ }^{*} \mathrm{a},{ }^{* *}$ & $\mathrm{c}^{*}$ \\
ABCD & $\mathrm{abc} \mathrm{c}^{*}$ & $\mathrm{abc}$ \\
\hline
\end{tabular}

Then, the matching algorithm goes through the following sequence of actions. Steps 1-16 do the initial exploration of the tree through to rejecting a full, but false, hypothesis. Steps 17-21 continue to explore the right subtree, looking for 
alternatives with the same size, until a wildcard match is found. This leads to a hypothesis with a size smaller than that currently requested. Since no more right subtree hypotheses have the desired size (because they are generated large-to-small and the most recent generation is smaller than requested), the algorithm generates a new left subtree match in steps 22-24. At step 25, the left subtree also has a smaller match than desired, so the algorithm then reduces the size of the desired goal. Steps 26-30 generate the new reduced size match. Here the algorithm starts with previously generated matches of the desired size. At step 31 , the full match occurs, and although it is smaller than the requested size $(2+2)$, it also satisfies the termination threshold (3), so the match terminates successfully.

\begin{tabular}{cccll}
\hline & Model & Size & & \\
Step & Level & Goal & Pairings & Action \\
\hline 1 & ABCD & $(2,2)$ & - & get left submodel \\
2 & AB & $(1,1)$ & - & get left submodel \\
3 & A & 1 & Aa & accept pairing (true) \\
4 & AB & $(1,1)$ & - & get right submodel \\
5 & B & 1 & Ba & reject bad pairing \\
6 & B & 1 & Bb & accept pairing (true) \\
7 & AB & $(1,1)$ & ABab & accept pairing (true) \\
8 & ABCD & $(2,2)$ & - & get right submodel \\
9 & CD & $(1,1)$ & - & get left submodel \\
10 & C & 1 & Ca & reject bad pairing \\
11 & C & 1 & Cb & reject bad pairing \\
12 & C & 1 & Cc & accept pairing (true) \\
13 & CD & $(1,1)$ & - & get right submodel \\
14 & D & 1 & Da & accept pairing (bad) \\
15 & CD & $(1,1)$ & CDca & accept pairing (bad) \\
16 & ABCD & $(2,2)$ & ABCDabca & reject bad pairing \\
17 & ABCD & $(2,2)$ & - & get right submodel \\
18 & CD & $(1,1)$ & - & get right submodel \\
19 & D & 1 & Db & reject bad pairing \\
20 & D & 1 & Dc & reject bad pairing \\
21 & D & 1 & D* & accept wildcard pairing \\
22 & CD & $(1,1)$ & CDc* & reduce pairing size attempt rejected \\
23 & CD & $(1,1)$ & - & get left submodel \\
24 & C & 1 & C* & accept wildcard pairing \\
25 & CD & $(1,1)$ & - & reduce pairing size accepted \\
& & & - & (1,0) or (0,1) possible \\
26 & CD & $(1,0)$ & - & restart and generate left \\
27 & C & 1 & Cc & retrieve old match \\
28 & CD & $(1,0)$ & - & restart and generate right \\
29 & D & 0 & D* & retrieve old match \\
30 & CD & $(1,0)$ & CDc* & accept pairing (true) \\
31 & ABCD & $(2,2)$ & ABCDabc* & early termination occurs \\
& & & &
\end{tabular}

